\title{
The NuSTAR Extragalactic Surveys: Unveiling Rare, Buried AGNs and Detecting the Contributors to the Peak of the Cosmic X-Ray Background
}

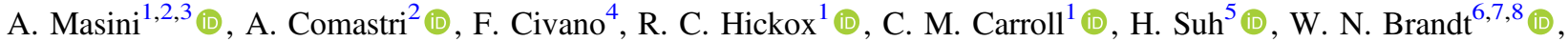 \\ M. A. DiPompeo ${ }^{1}$ (i), F. A. Harrison ${ }^{9}$, and D. Stern ${ }^{10}$ (1) \\ ${ }^{1}$ Department of Physics and Astronomy, Dartmouth College, 6127 Wilder Laboratory, Hanover, NH 03755, USA; alberto.masini@dartmouth.edu \\ 2 INAF-Osservatorio di Astrofisica e Scienza dello Spazio di Bologna, via Gobetti 93/3, I-40129 Bologna, Italy \\ 3 Dipartimento di Fisica e Astronomia (DIFA), Università di Bologna, via Gobetti 93/2, I-40129 Bologna, Italy \\ ${ }^{4}$ Harvard-Smithsonian Center for Astrophysics, 60 Garden Street, Cambridge, MA 02138, USA \\ ${ }^{5}$ Subaru Telescope, National Astronomical Observatory of Japan, 650 A'ohoku place, Hilo, HI, 96720, USA \\ ${ }^{6}$ Department of Astronomy and Astrophysics, 525 Davey Lab, The Pennsylvania State University, University Park, PA 16802, USA \\ 7 Institute for Gravitation and the Cosmos, The Pennsylvania State University, University Park, PA 16802, USA \\ ${ }^{8}$ Department of Physics, 104 Davey Laboratory, The Pennsylvania State University, University Park, PA 16802, USA \\ ${ }^{9}$ Cahill Center for Astronomy and Astrophysics, California Institute of Technology, Pasadena, CA 91125, USA \\ 10 Jet Propulsion Laboratory, California Institute of Technology, 4800 Oak Grove Drive, Pasadena, CA 91109, USA \\ Received 2018 July 20; revised 2018 August 31; accepted 2018 September 27; published 2018 November 12
}

\begin{abstract}
We report on the results of active galactic nuclei (AGNs) detection by NuSTAR performed in three extragalactic survey fields (COSMic Evolutionary Survey field (COSMOS), Ultra Deep Survey (UDS), and Extended Chandra Deep Field-South (ECDFS)) in three hard bands, namely H1 (8-16 keV), H2 (16-24 keV), and VH (35-55 keV). The aggregated area of the surveys is $\sim 2.7 \mathrm{deg}^{2}$. While a large number of sources is detected in the H1 band (72 at the $97 \%$ level of reliability), the $\mathrm{H} 2$ band directly probing close to the peak of the Cosmic X-ray Background (CXB) returns four significant detections, and two tentative (although not significant) detections are found in the VH-band. All of the sources detected above $16 \mathrm{keV}$ are also detected at lower energies. We compute the integral number counts for sources in such bands, which show broad consistency with population-synthesis models of the CXB. We furthermore identify two Compton-thick AGNs, one in the COSMOS field, associated with a hard and faint Chandra source, and one in the UDS field, never detected in the X-ray band before. Both sources are at the same redshift $z \sim 1.25$, which shifts their Compton-hump into the $\mathrm{H} 1$ band, and were previously missed in the usually employed NUSTAR bands, confirming the potential for using the H1 band to discover obscured AGNs at $z>1$ in deep surveys.
\end{abstract}

Key words: galaxies: active - galaxies: Seyfert

\section{Introduction}

X-ray surveys are one of the most effective ways to detect, select, and identify accreting supermassive black holes (see Brandt \& Alexander 2015, for a review). In the past decades, comprehensive X-ray surveys by XMM-Newton and Chandra covered a wide range in the flux-area plane, exploring a large range in redshift and luminosity, and characterizing the properties and evolution of active galactic nuclei (AGNs; Cappelluti et al. 2009; Elvis et al. 2009; Xue et al. 2011; Ranalli et al. 2013; Luo et al. 2017), which are the major contributors to the diffuse extragalactic emission named the Cosmic X-ray Background (CXB). The intensity of the CXB can be ascribed to a mixed contribution from all different kinds of AGNs (e.g., Setti \& Woltjer 1989; Comastri et al. 1995), showing a large range in obscuration, luminosity, and redshift. In particular, a nonnegligible contribution from a class of heavily obscured AGNs, called Compton-thick $\left(N_{\mathrm{H}}>10^{24}\right.$ $\mathrm{cm}^{-2}$ ), is required in order to successfully explain the intensity of the CXB around its peak at $\sim 20-30 \mathrm{keV}$ (e.g., Gilli et al. 2007; Treister et al. 2009; Draper \& Ballantyne 2010; Akylas et al. 2012).

Despite being very successful in detecting and describing the mixture of such different populations at low energies $(<10$ $\mathrm{keV}$ ), X-ray surveys are affected by a substantial absorption bias, mainly in the local universe $(z<1)$, where most of the effects of gas obscuration along the line of sight are seen.
Depending on the degree of obscuration, the intrinsic flux of a source can be significantly attenuated up to $\sim 10-20 \mathrm{keV}$, ultimately driving the source to be undetected in deep surveys. The effects of such obscuration become less significant at high energies, in particular in the hard X-ray band $(E>10 \mathrm{keV})$. However, hard X-ray surveys performed in the past years with coded-mask instruments like INTEGRAL and Swift-BAT detected a tiny fraction of the obscured sources making up the majority of the CXB above $10 \mathrm{keV}$ (Krivonos et al. 2005; Ajello et al. 2008).

With the advent of NuSTAR (Harrison et al. 2013), the first focusing hard X-ray telescope (comprising two focal plane modules, namely FPMA and FPMB), sensitive hard X-ray surveys above $10 \mathrm{keV}$ started to be feasible, allowing sources to be detected at $\sim 100 \times$ fainter fluxes with respect to codedmask instruments. A wedding-cake strategy for the NuSTAR surveys was adopted: a shallow, wide-area survey of the COSMic Evolutionary Survey field (COSMOS; Civano et al. 2015), a deep, pencil-beam survey of the Extended Chandra Deep Field-South (ECDFS; Mullaney et al. 2015), and a serendipitous survey (Alexander et al. 2013; Lansbury et al. 2017) were the first steps of a comprehensive survey program, which is now complemented by the observations of the Extended Groth Strip (J. Aird et al. 2018, in preparation), Chandra Deep Field-North (A. Del Moro et al. 2018, in preparation), and UKIDSS - Ultra Deep Survey (UDS; Masini et al. 2018) fields. 
Table 1

Details on the Single Fields Considered in This Work

\begin{tabular}{lrrrrl}
\hline \hline Field & $\begin{array}{r}\text { R.A. } \\
(\mathrm{deg})\end{array}$ & $\begin{array}{c}\text { Decl. } \\
(\mathrm{deg})\end{array}$ & $\begin{array}{c}\text { Area } \\
\left(\mathrm{deg}^{2}\right)\end{array}$ & $\begin{array}{l}T_{\text {exp }} \\
(\mathrm{ks})\end{array}$ & References \\
\hline COSMOS & 150.2 & 2.2 & 1.81 & 155 & Civano+15 \\
UDS & 34.4 & -5.1 & 0.58 & 345 & Masini+18 \\
ECDFS & 53.1 & -27.8 & 0.35 & 420 & Mullaney+15 \\
\hline
\end{tabular}

Note. The area column refers to the total area, while the exposure time $\left(T_{\exp }\right)$ column is the FPMA+FPMB exposure time at which the area drops to $0.01 \mathrm{deg}^{2}$.

Recently, Masini et al. (2018, M18 hereafter) presented the results of a NUSTAR survey of the UKIDSS-UDS field. In addition to adopting the three most commonly used energy bands (F: 3-24 keV, S: 3-8 keV, H: 8-24 keV), they explored the feasibility of source detection in three additional hard bands, splitting the commonly adopted hard band $(8-24 \mathrm{keV})$ in two bands $(8-16 \mathrm{keV}$ and $16-24 \mathrm{keV}, \mathrm{H} 1$ and $\mathrm{H} 2$ hereafter) and in a very-hard band (35-55 keV, VH hereafter), chosen as the energy interval where the NUSTAR background is mainly instrumental and well described by a fairly flat power law (Wik et al. 2014). Splitting the H-band into two sub-bands is important for two reasons. On one side, the background contribution is limited in the H1 band, allowing some sources to be more significantly detected narrowing the band; on the other hand, selecting sources at $\sim 16-24 \mathrm{keV}$ in the $\mathrm{H} 2$ band helps detecting directly those AGNs contributing the most to the peak of the CXB. ${ }^{11}$ The VH-band was chosen in order to exploit, for the first time in a deep extragalactic survey, the broad NUSTAR spectral coverage.

Few sources were detected in these bands, the majority of which were in the $\mathrm{H} 1$ band. Only one source was reliably detected in the $\mathrm{H} 2$ band, while no sources were detected in the VH-band. These results were expected, given the intensity and shape of the NUSTAR background and the survey area and depth, thanks to a large set of simulations, run in order to maximize the reliability and completeness of source detection at the same time (Civano et al. 2015, M18).

In this paper, we exploit the homogeneity of the NUSTAR survey strategy adopted in its extragalactic surveys program, by performing source detection in the $\mathrm{H} 1, \mathrm{H} 2$, and VH-bands also in the COSMOS and ECDFS fields, ultimately aggregating the results with those coming from the UDS field.

We assume a flat $\Lambda$ CDM cosmology $\left(H_{0}=70 \mathrm{~km} \mathrm{~s}^{-1} \mathrm{Mpc}^{-1}\right.$, $\Omega_{\mathrm{M}}=0.3, \Omega_{\Lambda}=0.7$ ) throughout the paper, which is organized as follows: the data sets and the sample are presented in Sections 2 and 3. We compute sensitivities in Section 4 and use them to obtain the integral number counts in Section 5. Section 6 presents an interesting source previously missed by NUSTAR and barely detected by Chandra in the COSMOS field as well as a source detected for the first time in the X-ray band in UKIDSS-UDS. We draw our conclusions in Section 8.

\footnotetext{
${ }^{11}$ The $\mathrm{H} 2$ band energy range was chosen to preserve the original width of the NuSTAR 8-24 keV band. Another possibility would have been to bridge the gap with the VH-band and define the $\mathrm{H} 2$ band as $\mathrm{H} 2=16-35 \mathrm{keV}$. It is not yet clear how the interplay between the larger band and the higher NuSTAR instrumental background above $\sim 25 \mathrm{keV}$ would impact the detection of hard sources in such a band.
}

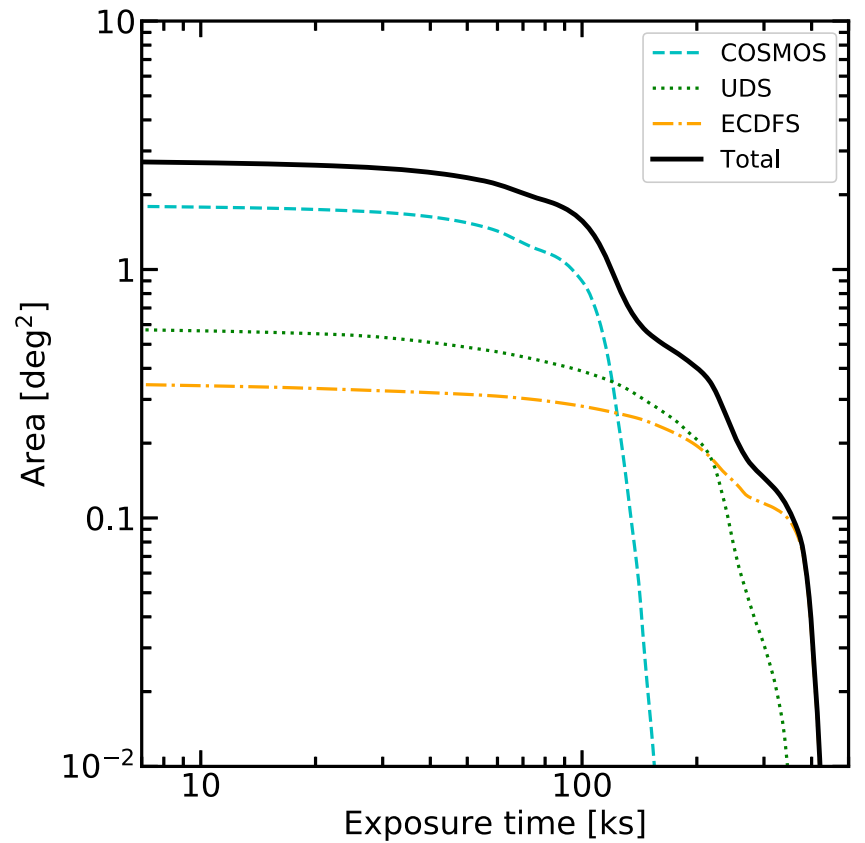

Figure 1. Area as a function of FPMA+FPMB exposure for the three field considered in this work (COSMOS, dashed cyan; UDS, dotted green; ECDFS, dot-dashed orange). The summed area curve is shown as the thick black line, for a total area of $2.74 \mathrm{deg}^{2}$.

\section{Data Sets}

We combine the NUSTAR surveys performed in three different fields (COSMOS, ECDFS, UDS; see Table 1) in order to maximize the total survey area. In Figure 1, the field areas (and the total one resulting from their sum) as a function of exposure are shown. Since creating the exposure maps in the $\mathrm{H} 1$ and $\mathrm{H} 2$ bands is time consuming, and the exposure maps in the NUSTAR $\mathrm{H}$ band were already available, we adopted the $\mathrm{H}$ band exposure maps for both the $\mathrm{H} 1$ and $\mathrm{H} 2$ bands, ${ }^{12}$ and created with the nuexpomap task the exposure maps in the VH-band only, weighted at $E=44.35 \mathrm{keV}$ (see Civano et al. 2015, and M18, for further details). The total area is 2.74 $\mathrm{deg}^{2}$, with a half-area depth of $108 \mathrm{ks}$, with the two NuSTAR focal plane modules (FPMA and FPMB) summed together.

Details about the single surveys are available in the appropriate papers. In particular, we follow the same strategy as M18 in order to make the analysis as homogeneous as possible. Refer to the same paper for details on the data analysis, background maps production, runs of simulations, and detection strategy. The DET_ML ${ }^{13}$ thresholds used in this work, corresponding to the $99 \%$ and $97 \%$ levels of reliability (defined through simulations as the ratio between sources matched with their counterpart, and detected sources, at a given DET_ML), and the number of sources detected above a given threshold in each band are reported in Table 2. For example, in the simulations run in the COSMOS field, $99 \%$ of the detected sources with a DET_ML $\geqslant 16.59$ were matched to their input counterparts (while the remaining 1\% was not matched, being made up of spurious sources).

\footnotetext{
12 Masini et al. (2018) have shown that adopting the $\mathrm{H}$ band exposure map for the $\mathrm{H} 1$ and $\mathrm{H} 2$ bands results in an underestimation of the exposure of at most $3 \%$, and overestimation of the exposure of at most $12 \%$, respectively.

${ }^{13}$ This quantity is defined as DET_ML $=-\ln (P)$, where $P$ is the Poissonian probability that the source is a background fluctuation. The higher the DET_ML, the higher the significance of a source.
} 
Table 2

Detection Thresholds and Numbers in Each Field and Band

\begin{tabular}{|c|c|c|c|c|c|c|c|c|c|c|c|c|}
\hline \multirow{5}{*}{ Field } & \multicolumn{12}{|c|}{ Reliability } \\
\hline & \multicolumn{6}{|c|}{ 99\% (Spurious Fraction: 1\%) } & \multicolumn{6}{|c|}{ 97\% (Spurious Fraction: 3\%) } \\
\hline & \multicolumn{6}{|c|}{ Bands } & \multicolumn{6}{|c|}{ Bands } \\
\hline & \multicolumn{2}{|c|}{$8-16 \mathrm{keV}$} & \multicolumn{2}{|c|}{$16-24 \mathrm{keV}$} & \multicolumn{2}{|c|}{$35-55 \mathrm{keV}$} & \multicolumn{2}{|c|}{$8-16 \mathrm{keV}$} & \multicolumn{2}{|c|}{$16-24 \mathrm{keV}$} & \multicolumn{2}{|c|}{$35-55 \mathrm{keV}$} \\
\hline & Thr. & $\mathrm{N}$ & Thr. & $\mathrm{N}$ & Thr. & $\mathrm{N}$ & Thr. & $\mathrm{N}$ & Thr. & $\mathrm{N}$ & Thr. & $\mathrm{N}$ \\
\hline COSMOS & 16.59 & 32 & 19.07 & 2 & 21.36 & 0 & 14.49 & 38 & 16.95 & 3 & 20.69 & 0 \\
\hline UDS & 15.13 & 16 & 17.54 & 1 & 23.55 & 0 & 13.23 & 20 & 16.09 & 1 & 23.00 & 0 \\
\hline ECDFS & 16.81 & 11 & 19.09 & 0 & 28.54 & 0 & 14.22 & 14 & 16.86 & 0 & 28.38 & 0 \\
\hline Tot & & 59 & & 3 & & 0 & & 72 & & 4 & & 0 \\
\hline
\end{tabular}

Note. The thresholds are expressed in terms of DET_ML, defined as DET_ML $=-\ln (P)$ where $P$ is the Poissonian probability that the source is a background fluctuation. The thresholds have been computed exploiting a large set of simulations, in order to keep under control the spurious fraction. See Civano et al. (2015) and M18 for further details.

\section{The Aggregated Sample}

As can be seen from the last row of Table 2, regardless of the reliability threshold, the $\mathrm{H} 2$ and $\mathrm{VH}$-bands return very few or no detections at all. In particular, of four sources significantly detected at the $97 \%$ level of reliability in the $\mathrm{H} 2$ band across the aggregated fields, no one is exclusively detected in the H2 band. The majority of these sources (three) come from the COSMOS field, which is the widest of the set, while none comes from ECDFS, the deepest one. This is probably due to the rather high NuSTAR background in the $\mathrm{H} 2$ band, which requires sources to be very bright (and henceforth rare) in order to be robustly detected.

Following M18, we focus on the largest sample, at the cost of a slightly lower reliability (spurious fraction of 3\%). The total sample of sources detected in at least one band is composed of 72 sources across the COSMOS (38 sources), UDS (20 sources), and ECDFS (14 sources) fields.

\subsection{Matches with Previous NuSTAR Catalogs}

Out of the 38 sources detected in the COSMOS field, 35 are matched with a counterpart from Civano et al. (2015) within $30^{\prime \prime}$. Such a matching radius has been adopted also in other NuSTAR Extragalactic Surveys (Civano et al. 2015; Mullaney et al. 2015, M18) and we refer the interested reader to those papers for more details. One of the three unmatched sources has its counterpart at a distance of $32^{\prime \prime}$, so slightly above our matching radius, and we consider this source as real, while the other two do not have a close counterpart. One of these two sources is significantly above the $99 \%$ of reliability threshold in the $\mathrm{H} 1$ band and may have been missed by Civano et al. (2015) due to the background contribution in the $\mathrm{H} 2$ band (in which it is undetected) lowering the source significance in the aggregated $8-24 \mathrm{keV}$ band. We are going to focus on this source in Section 6. The last one could be a spurious source, since its significance is just above the adopted threshold of $97 \%$ reliability. Indeed, from our spurious fraction of $3 \%$, we expect an average of $\approx 1$ sources to be a false detection in the COSMOS field.

Nineteen of the 20 sources detected in the UKIDSS-UDS field are matched to the catalog of M18. The only source missing a counterpart has a DET_ML just above the threshold and may be a spurious source as well. A discussion of the possible counterparts of this source is presented in Section 6.1.
Table 3

Balance between $\mathrm{H}$ and $\mathrm{H} 1$ Bands at the $99 \%$ Reliability Level in the Three Fields Considered

\begin{tabular}{llccc}
\hline \hline Field & H & H1 & In Common & Gained/Lost \\
\hline COSMOS & 32 & 32 & 26 & $+6 /-6$ \\
UDS & 15 & 16 & 13 & $+3 /-2$ \\
ECDFS & 19 & 11 & 11 & $0 /-8$ \\
\hline
\end{tabular}

Note. The gained/lost column refers to adopting the H1 band over the $\mathrm{H}$ one.

All the 14 sources detected in the ECDFS are matched to the catalog of Mullaney et al. (2015).

Given these results, one may think that the sources detected in the $\mathrm{H} 1$ band are the same ones detected in the broader $\mathrm{H}$-band, giving no reason to prefer the H-band over the H1 band. However, some differences appear at a closer look. Indeed, if the narrower band suffers from less background, it also loses sensitivity over the broader one. We stick to the $99 \%$ reliability threshold to have an easier and more homogeneous comparison with the UDS, COSMOS, and ECDFS catalogs. As can be seen from Table 3, there are 32 sources detected in the COSMOS field in the H-band, and 32 in the $\mathrm{H} 1$ band as well. However, only 26 of these are in common. In other words, employing the $\mathrm{H} 1$ band, six more sources are detected, but six sources are lost at the same time. Similarly, in UDS there are 15 sources detected in the H-band, and 16 in the $\mathrm{H} 1$ band. In this case, 13 sources are in common: preferring the $\mathrm{H} 1$ band, three sources are gained, but two sources are lost. In ECDFS, there are 19 sources in the H-band, and 11 in the $\mathrm{H} 1$ band. All $11 \mathrm{H} 1$ sources are detected in the H-band, implying a loss of eight sources.

We conclude that there is no clear benefit in employing the $\mathrm{H} 1$ band over the H-band in a deep NUSTAR survey, at least performing detections at the $99 \%$ reliability level. However, as we shall see in the following sections, there are few cases in which the $\mathrm{H} 1$ band is extremely helpful in selecting interesting sources with a particular spectral shape.

\subsection{Other Details of the Sample}

Our final sample is made of 72 sources. Every source in this sample is detected above the threshold of $97 \%$ reliability in the $\mathrm{H} 1 \mathrm{band}$; four of these sources are also significantly detected in the $\mathrm{H} 2$ band, while no sources are detected above the threshold 
in the VH-band. Two sources (id158 and id218 in the catalog of Civano et al. 2015) have a sub-threshold counterpart in the VH-band and are both detected in the COSMOS field. This means that a detection in the VH-band has been found for these two sources, albeit their DET_ML values in the VH-band are not sufficient to claim a detection at the $97 \%$ reliability level. In particular, the first one (DET_ML $L_{V H} \sim 11.66$ ) is associated with a quasar at $z=1.509$ (spectroscopic) and is detected above the threshold in the $\mathrm{H} 1$ band but undetected in the $\mathrm{H} 2$ band. The second one (DET_ML $\mathrm{VH}_{\mathrm{VH}} \sim 8.1$, associated with a quasar at a spectroscopic redshift of $z=0.345$ ) is robustly detected in both the hard sub-bands $\mathrm{H} 1$ and $\mathrm{H} 2$, giving further support to the hypothesis that the weak emission in the VH-band could be associated with such a bright source.

\section{Sensitivities}

In order to adequately take into account the survey sensitivity, we compute the completeness at the $97 \%$ reliability threshold for each field and each band. Further details on the definitions of reliability and completeness for our surveys are provided in Civano et al. (2015) and M18. We briefly recall here that they both exploit the large suite of simulations run for each energy band and each field. The reliability sets the threshold above which a source is considered to be real, keeping the spurious fraction under control. The completeness is computed as the ratio of the number of sources detected above a defined reliability threshold and matched to their counterparts, and the number of sources injected in a simulation, as a function of their input flux. In other words, at high fluxes, the completeness curve is unity, because bright sources are easily detected and matched to their input counterparts; at fainter fluxes, more and more sources are missed (and spurious sources are more easily detected), lowering the survey completeness. Rescaling this curve for the total area, a sensitivity curve is obtained, which naturally encompasses the Poissonian fluctuations of the background at low fluxes (e.g., Georgakakis et al. 2008).

This procedure has been adopted in an identical way for the three fields and for each band, ${ }^{14}$ and the total area curve is obtained by summing the sensitivity curves of the three fields. The sensitivities for the $\mathrm{H} 1, \mathrm{H} 2$, and $\mathrm{VH}$-bands are shown in Figure 2.

\section{5. $\log N-\log S$}

For every source and in each band, we extract total counts, background counts, and average exposures from a circular aperture of $20^{\prime \prime}$ from the data mosaics, background mosaics and exposure maps, respectively. Detections and non-detections are treated in the same way of Civano et al. (2015) and M18.

Following Harrison et al. (2016), we adopt a Bayesian method in calculating the number counts of our sources (Georgakakis et al. 2008). Briefly, instead of assuming a fixed flux (coming, e.g., from aperture photometry) with no uncertainty, we compute the probability density function for each source of having a flux $f$ inside a defined range of fluxes. The minimum flux is a factor of three lower than the flux limit at the $50 \%$ of completeness in each band as reported by M18, while the maximum flux is $10^{-12} \mathrm{erg} \mathrm{cm}^{-2} \mathrm{~s}^{-1}$ for the $\mathrm{H} 1$ and

\footnotetext{
${ }^{14}$ Due to the rarity of bright sources in the VH-band in the smallest fields (UDS and ECDFS), a total of 1000 simulations were run. We ran 400 simulations for the other bands, consistently with M18.
}
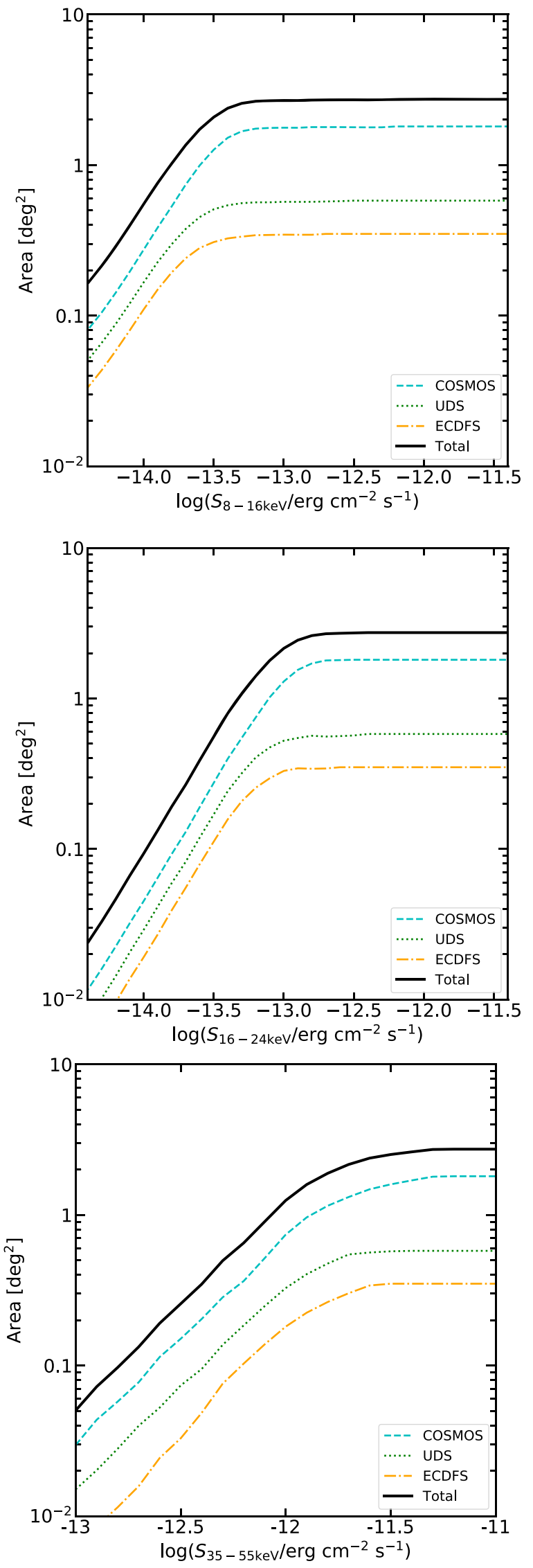

Figure 2. From top to bottom: sensitivity for the $\mathrm{H} 1, \mathrm{H} 2$, and $\mathrm{H} 3$ band, respectively. In each panel, the COSMOS curve is shown by the cyan dashed line, the UDS one in dotted green, and the ECDFS one in dotted-dashed orange. The total curve is the black thick line. 

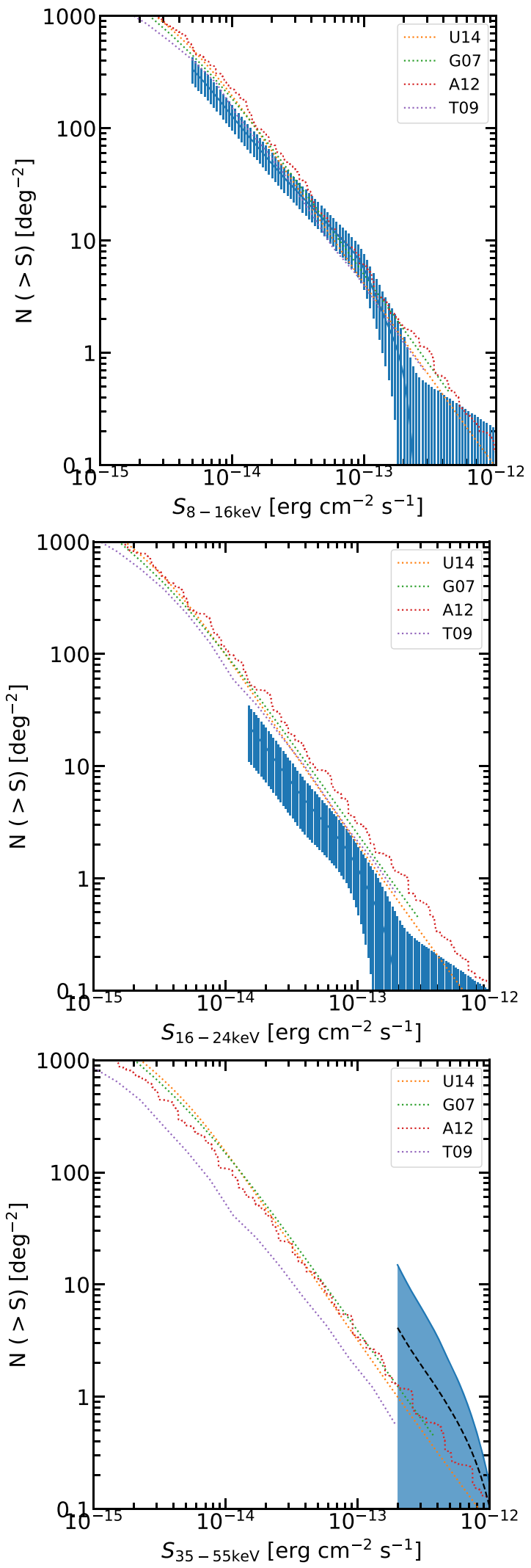

Figure 3. From top to bottom: $\log N-\log S$ for the H1, H2, and upper limits for the VH-band, compared with some population-synthesis models (in dotted lines: orange, Ueda et al. 2014; green, Gilli et al. 2007; red, Akylas et al. 2012; violet, Treister et al. 2009). The uncertainties are Poissonian and take into account a rough constant $13 \%$ of cosmic variance in the $\mathrm{H} 1$ band. In the bottom panel, the dashed black line denotes where the upper limit would lie adopting lower reliability thresholds, appropriate for the two tentative detections.
$\mathrm{H} 2$ bands, and $10^{-11} \mathrm{erg} \mathrm{cm}^{-2} \mathrm{~s}^{-1}$ for the VH-band. For each source, the expected number of total counts in order to have a flux $f$ is

$$
T=f t_{\exp } C \eta+B
$$

where $t_{\exp }$ is the exposure time of the source, $C$ is the conversion factor between fluxes and count rates (assuming an average photon index $\Gamma=1.8$; see, e.g., Burlon et al. 2011), $\eta$ is the factor to take into account the encircled energy fraction of the PSF (for NUSTAR and the aperture of $20^{\prime \prime}$ adopted to extract counts, this results in $\eta=0.32$; see Civano et al. 2015) and $B$ are the background counts. The probability of having $T$ total counts given the observed total counts $N$ is then (see Equation (6) in Georgakakis et al. 2008):

$$
P(f, N)=\frac{T^{N} e^{-T}}{N !} f^{\beta} .
$$

Here, $\beta$ is the slope of the differential number counts, and we assume $\beta=-2.81$ following Harrison et al. (2016). The exact choice of $\beta$ has a negligible impact on the slope of our integral number counts: varying $\beta$ by $40 \%$ results in a $3 \%$ variation of the integral number counts slope.

We normalize such density functions in order to have a unitary contribution of a source split on the whole flux range. Summing the single probability density functions for each source and dividing by the sensitivity curve gives the number counts. The integral number counts for sources detected by NuSTAR in the H1, H2, and VH-bands are shown in Figure 3, along with predictions from some population-synthesis models.

In the top panel of Figure 3, the cumulative number counts of our H1-detected sources is presented. Our Poisson uncertainties, which include a constant $\sim 13 \%$ of cosmic variance for the H1 band, estimated following Hickox \& Markevitch (2006), ${ }^{15}$ suggest that we compare broadly well with models. On the other hand, a comparison of our number counts in the $\mathrm{H} 2$ band with models (middle panel of Figure 3) shows that our data lie a factor of $\sim 2$ below the models' predictions. While this tension is mild, the systematic overestimation from models could suggest that NUSTAR is not detecting all of the $\mathrm{H} 2$ band sources it should, or that models predict too many sources to be detected in the $\mathrm{H} 2$ band. The very limited number of sources (four, above the threshold of $97 \%$ of reliability) prevents a more detailed discussion. Finally, assuming the signal in the VH-band to be real for the two COSMOS sources, we can place an upper limit on the number counts of sources in the VH-band. The upper limit shown in the bottom panel of Figure 3 is to be considered overestimated, since the two tentative detections have a significance lower than the threshold for which the sensitivity curve has been computed. As noted in Section 3.2, the two tentative detections have DET_ML values of $\sim 8.1$ and $\sim 11.6$. If we lower the reliability thresholds of the three fields, so that sources with DET_ML $\gtrsim 8.0$ are considered robust detections, the area seen at a given flux increases, at the cost of a lower reliability ( $\sim 20 \%-30 \%$ across the fields). The larger area surveyed at a given flux reflects in a lower value of the upper limit on the

\footnotetext{
${ }^{15} \mathrm{We}$ also validated our method exploiting the XMM-Newton Stripe 82 catalog (LaMassa et al. 2016). We measured the variance of sources extracted from randomly chosen circular areas of $2.7 \mathrm{deg}^{2}$, applying appropriate flux cuts in the $0.5-10 \mathrm{keV}$ band of $>3 \times 10^{-14} \mathrm{erg} \mathrm{cm}^{-2} \mathrm{~s}^{-1}$, broadly comparable with the NUSTAR limiting 8-16 keV flux of our detected sources, respectively.
} 

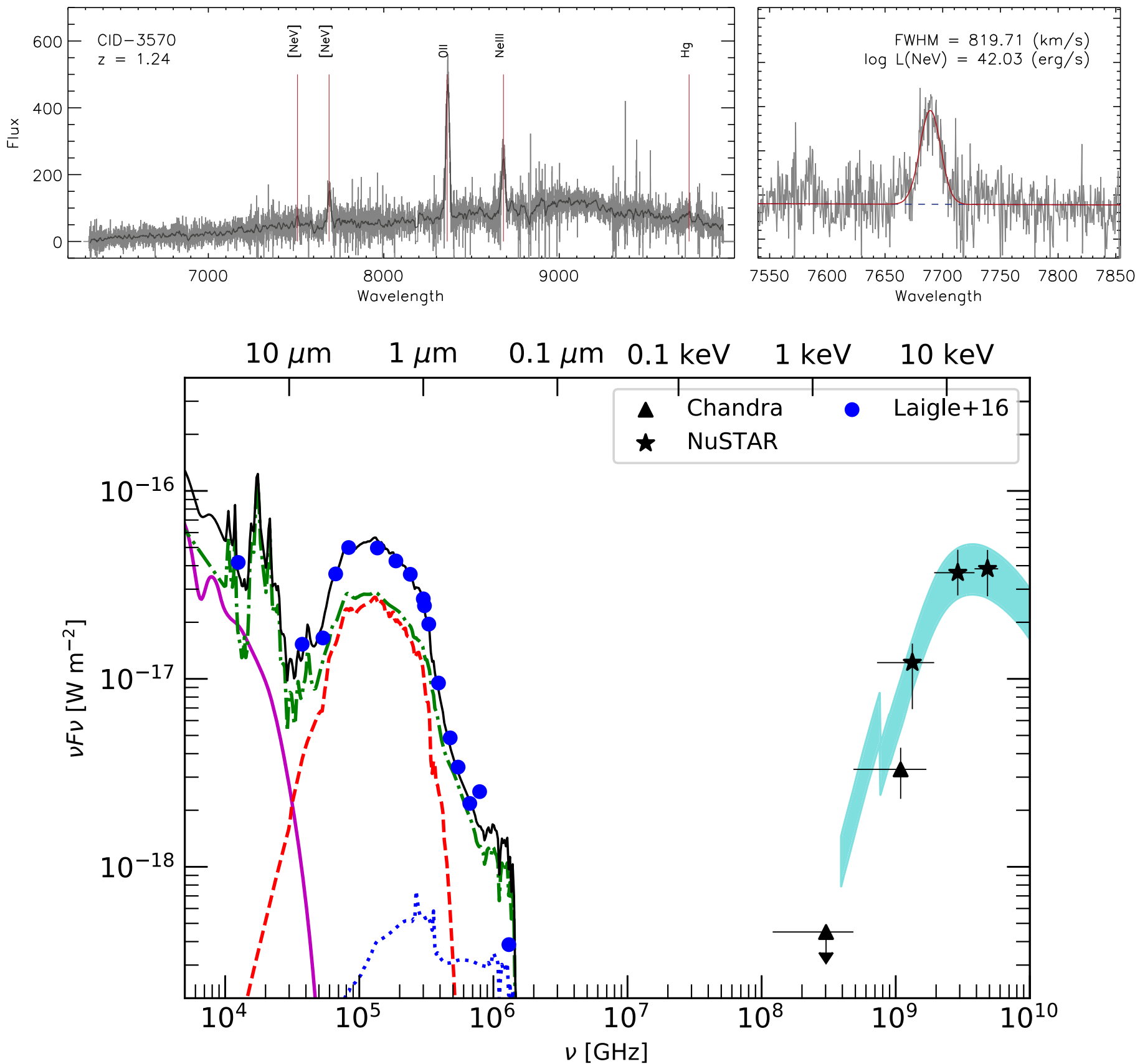

Figure 4. Top. DEIMOS spectrum of cid_3570 (Hasinger et al. 2018), with the right panel zooming on the Ne V emission line. Bottom: broadband SED for Cid_3570, the interesting source at $z=1.244$ in the COSMOS field significantly detected in the H1 band by NuSTAR. The optical-IR part of the SED is fitted with an AGN (solid violet, Assef et al. 2010), passive (dashed red, Assef et al. 2010), late-type (dotted-dashed green, Kirkpatrick et al. 2015), and star-forming (dotted blue, Assef et al. 2010) templates. Data points (blue circles) are from the catalog of Laigle et al. (2016), and references therein. In the X-ray part of the SED, the Chandra (triangles) soft (0.5-2 keV) band flux is an upper limit. The NuSTAR fluxes derived from the spectral analysis are labeled with stars. Uncertainties on $\nu F \nu$ are at $1 \sigma$ confidence level, and the cyan region is the pexrav (Magdziarz \& Zdziarski 1995) model $( \pm 1 \sigma)$ adopted to derive the NuSTAR fluxes.

integral number counts, indicated in the bottom panel of Figure 3 by the dashed black line.

\section{A Buried AGN in the COSMOS Field}

As already discussed in Section 3.1, one of the two sources in the COSMOS field that is not matched with the catalog of Civano et al. (2015) is above the threshold of $99 \%$ of reliability in the $\mathrm{H} 1$ band, and as a consequence, it would have also been detected in the most conservative sample of 59 sources, where the spurious fraction is $\sim 1 \%$ (see Table 2). While missing a previously detected NuSTAR counterpart, ${ }^{16}$ a faint Chandra source is found at $\sim 7^{\prime \prime}$ distance in the Chandra COSMOS Legacy Survey (cid_3570in the catalog of Civano et al. 2016). Chandra detected the source with $\sim 16$ full $(0.5-7 \mathrm{keV})$ band net counts, of which $\sim 13$ are in the hard (2-7 keV) band. As a consequence, this source has an hardness ratio $\gtrsim 0.68$ (Civano et al. 2016). In the optical band, cid_3570is

\footnotetext{
${ }^{16}$ We note that a source at $13^{\prime \prime}$ from our $N U S T A R$ position is detected by Civano et al. (2015) in the full and hard NUSTAR bands. However, being below the $99 \%$ reliability threshold, it was not included in the final catalog.
} 

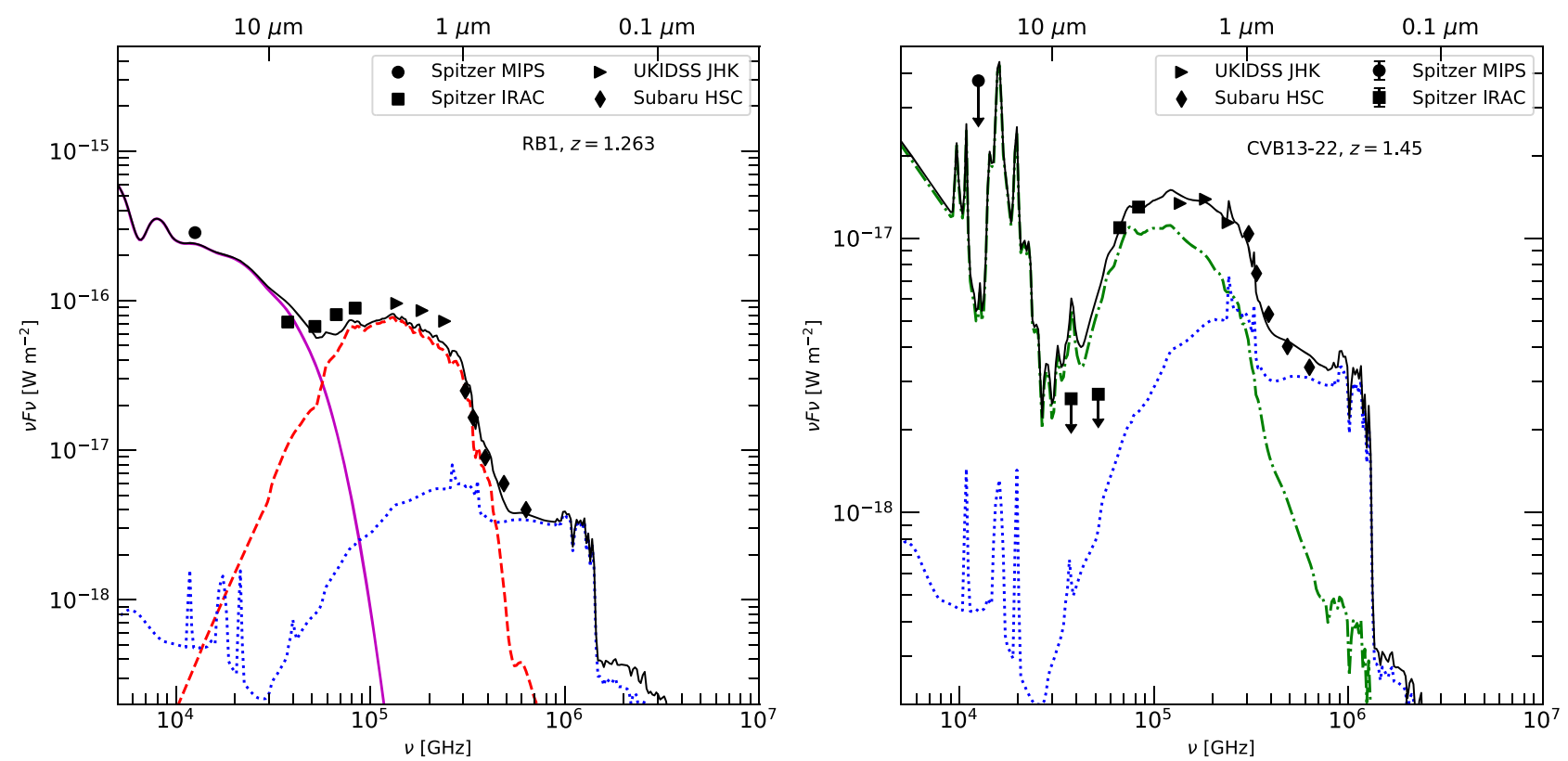

Figure 5. Optical/IR SEDs of RB1 (left) and CVB13-22 (right), the two possible counterparts to the NuSTAR detection in the UDS field. The circular data point is Spitzer MIPS, the square points refer to Spitzer IRAC, the rightward triangles to UKIDSS in the JHK bands, and the diamonds to Subaru HSC in the $g, r, i, z$, $Y$ bands. The optical-IR part of the SED is fitted with an AGN (solid violet), passive (dashed red), late-type (dotted-dashed green) and star-forming (dotted blue) templates. Upper limits are labeled as downward arrows and have been estimated considering the minimum flux of the sources detected by one instrument in the surroundings of the target. While the AGN component in the SED in RB1 is heavily reddened $(E(B-V) \sim 6.2)$, giving support to the classification of obscured (possibly CT) AGN, the same component in CVB13-22 is not required by the fit.

associated with a galaxy at redshift $z=1.244$ (Kartaltepe et al. 2015; Marchesi et al. 2016a). We thus expect the NuSTAR detection to be real, and associated with a highly obscured AGN with a hard spectrum barely detected by Chandra and previously missed by $N U S T A R$. The NUSTAR spectrum of this source is indeed very hard, and a fit with a power law returns a flat photon index $\left(\Gamma=0.56_{-1.03}^{+0.96}\right)$, and a default MYTorus ${ }^{17}$ model (Murphy \& Yaqoob 2009), assuming a photon index $\Gamma=1.8$, gives a column density $N_{\mathrm{H}}=2.3_{-1.7}^{+u} \times 10^{24} \mathrm{~cm}^{-2}$ at the $90 \%$ confidence limit. We notice that a pure reflection model (pexrav; Magdziarz \& Zdziarski 1995) also gives a good fit, statistically indistinguishable from the MYTorus model adopted to derive an estimate on the column density. In particular, the photon index is fixed to $\Gamma=1.8$, and the reflection parameter is fixed to a negative value in order to have a pure reflection component. All the other parameters are left frozen to their standard values. With this configuration, the only free parameter of the pexrav model is the normalization at $1 \mathrm{keV}$, which is $N=2.6_{-1.2}^{+1.4} \times 10^{-5}$ photons $\mathrm{keV} / \mathrm{cm}^{2} / \mathrm{s}$. Leaving the photon index free to vary does not improve the fit, and results in $\Gamma=1.4 \pm 1.0$. The DEIMOS (Faber et al. 2003) spectrum of cid_3570 (Hasinger et al. 2018) is shown in the top panels of Figure 4, where a clear [Ne V]3426 emission line can be seen, leaving no doubts on the presence of an AGN. The X-ray to $[\mathrm{Ne} \mathrm{V}]$ luminosity ratio $\left(L_{\mathrm{X}} / L_{[\mathrm{NeV}]} \sim 17\right)$, and the column density measured from the spectral analysis place cid_3570 in very good agreement with the expected trend of the $\mathrm{X} / \mathrm{Ne} \mathrm{V}$ ratio identified by Gilli et al. (2010) using a sample of local objects.

\footnotetext{
17 The parameter describing the column density $N_{\mathrm{H}}$ in this model is in the range $10^{22}-10^{25} \mathrm{~cm}^{-2}$; as such, if a measurement of $N_{\mathrm{H}}$ hits one of the caps, a letter $+u$ or $-l$ in the uncertainty is adopted for upper and lower caps, respectively.
}

In order to have a better view of the properties of cid_3570, we collected optical and near-IR data between $0.44 \mu \mathrm{m}$ and $24 \mu \mathrm{m}$, from the catalog of Laigle et al. (2016). In particular, we use data from GALEX (NUV), CFHT $(u)$, the Hyper Suprime Cam Subaru survey $\left(\mathrm{B}, \mathrm{V}, r, i^{+}, z^{++}, \mathrm{Y}\right)$, UltraVISTA (Y, J,H,K), and Spitzer (IRAC and MIPS 24). We also add information in the X-ray band coming from Chandra (0.5-2 keV and 2-7 keV; see Civano et al. 2016; Marchesi et al. 2016a, 2016b) and NuSTAR (S, H1, and H2 bands). Chandra fluxes are rescaled based on the count rate from Civano et al. (2016), assuming the best-fitting pexrav model and simulating a fake Chandra spectrum with the appropriate response and ancillary files (Marchesi et al. 2016b). NuSTAR fluxes are computed through spectral analysis and adopting the reflection (pexrav) model, and would be the same within the uncertainties adopting the MYTorus model. We show the Spectral Energy Distribution (SED) in the bottom panel of Figure 4 where the optical-IR part of the SED is fitted with a combination of four templates (C. M. Carroll et al. 2018, in preparation): one for the AGN (Assef et al. 2010), and three for the host galaxy (Assef et al. 2010; Kirkpatrick et al. 2015). Through a $\chi^{2}$ minimization, the software returns the best fit among all the possible combinations of the four templates. The X-ray part of the SED, on the other hand, is fitted with the pexrav model described before.

As can be seen from Figure 4, the AGN component is required to better fit the MIPS $24 \mu \mathrm{m}$ and IRAC $8 \mu \mathrm{m}$ points only, and as a consequence is extremely reddened $(E(B-V) \sim$ 22.3).

On the other hand, the inclusion of a cold dust component (Mullaney et al. 2011) in the fitting (e.g., Suh et al. 2017), due to the source being detected in the FIR band at $250 \mu \mathrm{m}$, may reduce the AGN contribution at $24 \mu \mathrm{m}$, and its relative reddening $(E(B-V) \sim 0.3)$. 
Such different results, dependent on the models adopted, point toward a general lack of evidence of the AGN component in the UV/optical/IR SED of cid_3570, giving on the other hand more importance to the X-ray detection. Indeed, regardless of the exact details of the SED fitting, the main point of this analysis is that the NUSTAR detection in the H1 band is associated with a real, highly obscured AGN. This motivates a deeper look to the unassociated source found in the UDS field as well.

\subsection{Another Buried AGN in UKIDSS-UDS?}

Motivated by the successful confirmation of a Comptonthick AGN for the unassociated source in the COSMOS field cid_3570, we focused on the source not matched with the catalog of M18 in the UDS field.

Within $30^{\prime \prime}$ from the $\mathrm{H} 1$ band position, we found one faint Chandra source (Kocevski et al. 2018) at a distance of $\sim 12^{\prime \prime}$, associated with a galaxy in a high-redshift cluster (namely CVB13-22, at spectroscopic redshift $z=1.4548$; see van Breukelen et al. 2007).

On the other hand, at approximately the same distance $\left(\sim 12^{\prime \prime}\right)$, we find a second possible counterpart to the NuSTAR detection. Indeed, Simpson et al. (2012) classify the source RB1 in van Breukelen et al. (2007) as a narrow line AGN at spectroscopic redshift $z=1.263$. The classification comes from Chuter et al. (2011) and is due to the detection of strong, high-ionization UV emission lines like C IV and [Ne III]. The steep NIR-MIR slope in the SED of RB1, contrary to the nondetection at the same wavelength of CVB13-22 (see Figure 5) argues in favor of this AGN being the correct counterpart, although we cannot rule out the possibility that the CVB13-22 source in the high-redshift cluster could also be at least partially contributing to the NUSTAR flux. If RB1 is the correct or dominant counterpart, the NUSTAR detection would be the first X-ray detection of this AGN. In either case, we also consider this detection to be real, which gives further support to the use of the $\mathrm{H} 1$ band as a tool to unveil candidate buried AGNs previously missed by X-ray surveys.

\section{Future Prospects for the $\mathrm{H} 1$ and $\mathrm{H} 2$ Bands}

As already discussed (Section 3.1), the H1 band is suitable to find interesting, rare, and obscured AGNs, but misses some of the H-band selected sources, due to the loss of sensitivity narrowing the band. Of a total of $72 \mathrm{H} 1$-band sources detected, three were not matched to any NUSTAR source previously detected in the F-, S-, or H-bands. Two out of three turned out to have an optical counterpart, showing signatures of buried AGNs at $z>1$. It is very likely that cid_3570 in COSMOS (Section 6) would have been robustly detected in the usual F and H-bands by Civano et al. (2015) relaxing the reliability threshold. On the other hand, RB1 in the UDS field (Section 6.1) was missed by M18, who even employed the less-conservative thresholds for the F-, S-, and H-bands. Given the large overlap with the $\mathrm{H}$ band selected sources, we suggest to employ the $\mathrm{H} 1$ band for follow-up observations of IRselected potentially buried AGN.

Regarding the four sources detected in the $\mathrm{H} 2$ band, they have broadly the same $\mathrm{H} 2$ band flux $\left(F_{\mathrm{H} 2} \sim 1-2 \times\right.$ $\left.10^{-13} \mathrm{erg} \mathrm{cm}^{-2} \mathrm{~s}^{-1}\right)$. At this flux, the total area is $\sim 2.45 \mathrm{deg}^{2}$, with an average exposure time $\sim 40 \mathrm{ks}$ (FPMA+FPMB). This implies that we found $\sim 1.6 \mathrm{H} 2$ band sources $/ \mathrm{deg}^{2}$, or equivalently one $\mathrm{H} 2$ band source for each $\sim 0.63 \mathrm{deg}^{2}$. This number is remarkably consistent with the one found in UDS $\left(\sim 0.6 \mathrm{deg}^{2}\right)$, three in COSMOS $\left(\sim 1.8 \mathrm{deg}^{2}\right)$, and none in ECDFS $\left(\sim 0.4 \mathrm{deg}^{2}\right)$. These numbers thus imply that NuSTAR is able to detect in the $\mathrm{H} 2$ band one source every $\sim 23$ pointings of $20 \mathrm{ks}$, if they are not overlapping. If overlapping with the half-FOV strategy commonly adopted in NuSTAR surveys, $50 \%$ more pointings are needed, requiring $\sim 34$ half-FOV shifted pointings to detect one source. Indeed, COSMOS is made up of 121 partially overlapping pointings, and should contain $\sim 3.6$ sources; UDS is made up of 35 half-FOV shifted pointings, just enough to detect one source. This is not happening for the ECDFS survey, which has two totally overlapping passes on a half-FOV shifted $4 \times 4$ square. The ever-growing NuSTAR Seredipitous Survey (Alexander et al. 2013; Lansbury et al. 2017), with its $\sim 12.5 \mathrm{deg}^{2}$ coverage at the minimum flux of our sources, should contain $\sim 20$ sources detectable in the $\mathrm{H} 2$ band by NuSTAR in $\sim 20 \mathrm{ks}$.

\section{Conclusions}

In this paper, we presented the aggregated results coming from three different NuSTAR survey fields in three hard bands $(\mathrm{H} 1$ : 8-16 keV, H2: 16-24 keV, VH: 35-55 keV), covering a total area of $2.7 \mathrm{deg}^{2}$. The main results can be summarized as follows:

1. Following the same strategy delineated in M18, we detected 72 sources above the $97 \%$ level of reliability in at least one band across three fields. All 72 sources are robustly detected in the $8-16 \mathrm{keV}$ band, while four of them are also detected in the 16-24 keV band and two are perhaps detected (albeit under-threshold) in the $35-55 \mathrm{keV}$ band. The expected spurious fraction of the aggregated sample is $3 \%$.

2. We computed the number counts for our sources. We took into account the Eddington bias in our sample, computing for each source a probability density function over a range of fluxes, i.e., allowing each source to contribute to each flux bin following its probability density function. A comparison with AGN populationsynthesis models shows broad consistency with our results, mainly in the $\mathrm{H} 1$ band. A tension of a factor of $\sim 2$ in the $\mathrm{H} 2$ band is seen between the data and the models. Upper limits are provided in the VH-band, assuming the two sub-threshold detections to be real.

3. Narrowing the NUSTAR hard (8-24 keV) band and employing the $\mathrm{H} 1$ band can help to select interesting and obscured sources previously missed due to the high background rising above $\sim 15 \mathrm{keV}$. We found at least one such example of a buried, likely Compton-thick AGN in the COSMOS field at $z \sim 1.25$, together with an elusive AGN in the UDS field. Despite the presence of the [Ne V] line and the NUSTAR spectral analysis, the SED of the source in COSMOS does not show strong evidence for the presence of and AGN. On the other hand, the most likely counterpart of the UDS detection shows a significant AGN component in its optical-IR SED.

4. Based on the results obtained in the $\mathrm{H} 2$ band, we computed that one $\mathrm{H} 2$ band source is detected every $\sim 0.6 \mathrm{deg}^{2}$, or equivalently every $\sim 23$ non-overlapping NuSTAR pointings. We predict that the NuSTAR Serendipitous Survey should contain $\sim 20$ H2 band sources robustly detectable by $N U S T A R$ in a minimum time of $20 \mathrm{ks}$. 
We thank the anonymous referee, whose comments helped improve the quality and clarity of the paper. This work was supported under NASA Contract NNG08FD60C, and made use of data from the NUSTAR mission, a project led by the California Institute of Technology, managed by the Jet Propulsion Laboratory, and funded by the National Aeronautics and Space Administration. We thank the NuSTAR Operations, Software, and Calibration teams for support with the execution and analysis of these observations. This research made use of the NUSTAR Data Analysis Software (NuSTARDAS) jointly developed by the ASI Science Data Center (ASDC, Italy) and the California Institute of Technology (USA). This research has also made use of data obtained from the Chandra Data Archive and the Chandra Source Catalog, and software provided by the Chandra X-ray Center (CXC). Figure 4 is partially based on data products from observations made with ESO Telescopes at the La Silla Paranal Observatory under ESO programme ID 179.A-2005 and on data products produced by TERAPIX and the Cambridge Astronomy Survey Unit on behalf of the UltraVISTA consortium. The DEIMOS spectrum presented herein was obtained at the W. M. Keck Observatory, which is operated as a scientific partnership among the California Institute of Technology, the University of California and the National Aeronautics and Space Administration. The Observatory was made possible by the generous financial support of the W. M. Keck Foundation. This research made use of Astropy, a community-developed core Python package for Astronomy (Astropy Collaboration et al. 2013; The Astropy Collaboration et al. 2018), and XSPEC (Arnaud 1996).

A.M. and A.C. acknowledge support from the ASI/INAF grant I/037/12/0011/13. R.C.H. acknowledges support from the National Science Foundation through CAREER award 1554584, and from NASA through grant number NNX15AP24G. W.N.B. acknowledges support from Caltech NuSTAR subcontract 44A1092750.

Facilities: NuSTAR, Chandra.

Software: NuSTARDAS, FTOOLS.

\section{ORCID iDs}

A. Masini (i) https://orcid.org/0000-0002-7100-9366

A. Comastri (1) https://orcid.org/0000-0003-3451-9970

R. C. Hickox (iD https://orcid.org/0000-0003-1468-9526

C. M. Carroll (1) https://orcid.org/0000-0003-3574-2963

H. Suh (i) https://orcid.org/0000-0002-2536-1633

W. N. Brandt (i) https://orcid.org/0000-0002-0167-2453

M. A. DiPompeo (iD https://orcid.org/0000-0001-6788-1701

D. Stern (iD https://orcid.org/0000-0003-2686-9241

\section{References}

Ajello, M., Greiner, J., Sato, G., et al. 2008, ApJ, 689, 666

Akylas, A., Georgakakis, A., Georgantopoulos, I., Brightman, M., \& Nandra, K. 2012, A\&A, 546, A98

Alexander, D. M., Stern, D., Del Moro, A., et al. 2013, ApJ, 773, 125

Arnaud, K. A. 1996, in ASP Conf. Ser. 101, Astronomical Data Analysis Software and Systems V, ed. G. H. Jacoby \& J. Barnes (San Francisco, CA: ASP), 17

Assef, R. J., Kochanek, C. S., Brodwin, M., et al. 2010, ApJ, 713, 970

Astropy Collaboration, Robitaille, T. P., Tollerud, E. J., et al. 2013, A\&A, 558, A33

Brandt, W. N., \& Alexander, D. M. 2015, A\&A Rv, 23, 1

Burlon, D., Ajello, M., Greiner, J., et al. 2011, ApJ, 728, 58

Cappelluti, N., Brusa, M., Hasinger, G., et al. 2009, A\&A, 497, 635

Chuter, R. W., Almaini, O., Hartley, W. G., et al. 2011, MNRAS, 413, 1678

Civano, F., Hickox, R. C., Puccetti, S., et al. 2015, ApJ, 808, 185

Civano, F., Marchesi, S., Comastri, A., et al. 2016, ApJ, 819, 62

Comastri, A., Setti, G., Zamorani, G., \& Hasinger, G. 1995, A\&A, 296, 1

Draper, A. R., \& Ballantyne, D. R. 2010, ApJL, 715, L99

Elvis, M., Civano, F., Vignali, C., et al. 2009, ApJS, 184, 158

Faber, S. M., Phillips, A. C., Kibrick, R. I., et al. 2003, Proc. SPIE, 4841, 1657

Georgakakis, A., Nandra, K., Laird, E. S., Aird, J., \& Trichas, M. 2008, MNRAS, 388, 1205

Gilli, R., Comastri, A., \& Hasinger, G. 2007, A\&A, 463, 79

Gilli, R., Vignali, C., Mignoli, M., et al. 2010, A\&A, 519, A92

Harrison, F. A., Aird, J., Civano, F., et al. 2016, ApJ, 831, 185

Harrison, F. A., Craig, W. W., Christensen, F. E., et al. 2013, ApJ, 770, 103

Hasinger, G., Capak, P., Salvato, M., et al. 2018, ApJ, 858, 77

Hickox, R. C., \& Markevitch, M. 2006, ApJ, 645, 95

Kartaltepe, J. S., Sanders, D. B., Silverman, J. D., et al. 2015, ApJL, 806, L35

Kirkpatrick, A., Pope, A., Sajina, A., et al. 2015, ApJ, 814, 9

Kocevski, D. D., Hasinger, G., Brightman, M., et al. 2018, ApJS, 236, 48

Krivonos, R., Vikhlinin, A., Churazov, E., et al. 2005, ApJ, 625, 89

Laigle, C., McCracken, H. J., Ilbert, O., et al. 2016, ApJS, 224, 24

LaMassa, S. M., Urry, C. M., Cappelluti, N., et al. 2016, ApJ, 817, 172

Lansbury, G. B., Stern, D., Aird, J., et al. 2017, ApJ, 836, 99

Luo, B., Brandt, W. N., Xue, Y. Q., et al. 2017, ApJS, 228, 2

Magdziarz, P., \& Zdziarski, A. A. 1995, MNRAS, 273, 837

Marchesi, S., Civano, F., Elvis, M., et al. 2016a, ApJ, 817, 34

Marchesi, S., Lanzuisi, G., Civano, F., et al. 2016b, ApJ, 830, 100

Masini, A., Civano, F., Comastri, A., et al. 2018, ApJS, 235, 17

Mullaney, J. R., Alexander, D. M., Goulding, A. D., \& Hickox, R. C. 2011, MNRAS, 414, 1082

Mullaney, J. R., Del-Moro, A., Aird, J., et al. 2015, ApJ, 808, 184

Murphy, K. D., \& Yaqoob, T. 2009, MNRAS, 397, 1549

Ranalli, P., Comastri, A., Vignali, C., et al. 2013, A\&A, 555, A42

Setti, G., \& Woltjer, L. 1989, A\&A, 224, L21

Simpson, C., Rawlings, S., Ivison, R., et al. 2012, MNRAS, 421, 3060

Suh, H., Civano, F., Hasinger, G., et al. 2017, ApJ, 841, 102

The Astropy Collaboration, Price-Whelan, A. M., Sipőcz, B. M., et al. 2018 , arXiv: 1801.02634

Treister, E., Urry, C. M., \& Virani, S. 2009, ApJ, 696, 110

Ueda, Y., Akiyama, M., Hasinger, G., Miyaji, T., \& Watson, M. G. 2014, ApJ, 786, 104

van Breukelen, C., Cotter, G., Rawlings, S., et al. 2007, MNRAS, 382, 971

Wik, D. R., Hornstrup, A., Molendi, S., et al. 2014, ApJ, 792, 48

Xue, Y. Q., Luo, B., Brandt, W. N., et al. 2011, ApJS, 195, 10 